\title{
Using UAV-Based Systems to Monitor Air Pollution in Areas with Poor Accessibility
}

\author{
Oscar Alvear, ${ }^{1,2}$ Nicola Roberto Zema, ${ }^{3}$ Enrico Natalizio, ${ }^{3}$ and Carlos T. Calafate ${ }^{1}$ \\ ${ }^{1}$ Department of Computer Engineering, Universitat Politècnica de València, Camino de Vera, S/N, 46022 Valencia, Spain \\ ${ }^{2}$ Department of Electrical Engineering, Electronics and Telecommunications, Universidad de Cuenca, Av. 12 de Abril, S/N, \\ Cuenca, Ecuador \\ ${ }^{3}$ Laboratoire Heudiasyc, Sorbonne Universités, Université de Technologie de Compiègne, CNRS, 57 Avenue de Landshut, \\ CS 60319, 60203 Compiegne Cedex, France \\ Correspondence should be addressed to Oscar Alvear; osal@doctor.upv.es
}

Received 8 March 2017; Revised 16 June 2017; Accepted 9 July 2017; Published 7 August 2017

Academic Editor: Guizhen Yu

Copyright (C) 2017 Oscar Alvear et al. This is an open access article distributed under the Creative Commons Attribution License, which permits unrestricted use, distribution, and reproduction in any medium, provided the original work is properly cited.

\begin{abstract}
Air pollution monitoring has recently become an issue of utmost importance in our society. Despite the fact that crowdsensing approaches could be an adequate solution for urban areas, they cannot be implemented in rural environments. Instead, deploying a fleet of UAV s could be considered an acceptable alternative. Embracing this approach, this paper proposes the use of UAVs equipped with off-the-shelf sensors to perform air pollution monitoring tasks. These UAVs are guided by our proposed Pollution-driven UAV Control (PdUC) algorithm, which is based on a chemotaxis metaheuristic and a local particle swarm optimization strategy. Together, they allow automatically performing the monitoring of a specified area using UAVs. Experimental results show that, when using PdUC, an implicit priority guides the construction of pollution maps by focusing on areas where the pollutants' concentration is higher. This way, accurate maps can be constructed in a faster manner when compared to other strategies. The PdUC scheme is compared against various standard mobility models through simulation, showing that it achieves better performance. In particular, it is able to find the most polluted areas with more accuracy and provides a higher coverage within the time bounds defined by the UAV flight time.
\end{abstract}

\section{Introduction}

Industrial growth has brought unforeseen technological advances to our societies. Unfortunately, the price to pay for these advances has been an increase of air pollution worldwide, affecting both our health [1] and our lifestyle.

Air quality monitoring is relevant not only for the people living in urban areas, but also because it directly affects crops and different animals/insects in rural environments [2]. Thus, different solutions for measuring air quality should be sought for such environments.

For abovementioned reasons, environmental organizations and governmental institutions are beginning to consider the monitoring of environmental pollutants as a primary goal $[3,4]$.

The majority of methods used insofar to keep track of air pollution in major cities rely on fixed monitoring stations [5].
However, the use of such dedicated architectures and hardware for pollution monitoring is outmatched, in theory, by the use of crowdsensing [6] in areas with a high population density. Also, new ground-vehicle-based mobile sensors, which would theoretically be able to cover the same areas as the fixed solutions while employing a reduced number of agents, are emerging as a viable alternative (e.g., $[7,8])$.

With respect to the widespread use of small pollution monitoring sensors embedded in mobile vehicles, the possible scenarios can be divided into two main classes:

(i) Urban environments, where it is possible to embed the sensors on a wide set of vehicles like bicycles $[9,10]$ or cars [8].

(ii) Rural and industrial areas, where vehicular traffic is scarce and limited to the main transportation arteries. 
In the latter case, crowdsensing often fails to provide enough data to obtain realistic measurements having the required granularity.

Regarding crowdsensing approaches, projects like [1113] relied on crowdsensing solutions to monitor pollution in urban areas. However, in rural and industrial zones, available options are quite more limited. In the particular case of large rural or industrial areas, a fleet of mobile vehicles could be efficiently used to cover the vast distances associated with them. Furthermore, the use of autonomous sensor carriers is even more encouraged in this case due to the following considerations:

(i) The relative absence of civilian population to be taken care of during robotic operations.

(ii) Stable and regulated positioning of obstacles.

(iii) Fewer constraints concerning UAV flight laws

(iv) Safety and security concerns, as some areas could be dangerous to access for human operators.

Since, in these environments, ground access is usually hindered and full of obstacles, the most feasible way to implement a fleet of mobile pollution monitoring robots is via Unmanned Aerial Vehicles (UAVs) [14].

Taking the aforementioned issues into consideration, in this paper we propose the use of UAVs equipped with commercial and off-the-shelf (COTS) devices and sensors to implement a service of air pollution monitoring that leverages the use of bioinspired approaches as its main control strategy. These choices allow covering a specific area automatically and enable discovering the pollution distribution of a large area by prioritizing the most polluted zones inside it.

We show that, using our chemotaxis-based approach for UAV path control, it is possible to achieve faster and more accurate estimations about the location of the most polluted areas with respect to classical area-search approaches. Our analysis also takes into account uncertainty-based considerations in the sensor sampling operations.

This paper is organized as follows: in Section 2 we refer to some related works addressing UAV-based sensing, UAV mobility models, and UAV control protocols. Sections 3 and 4 present an overview of the UAV Configuration and the UAV Control System, respectively. In Section 5, we compare our algorithm against the Billiard and Spiral mobility models via simulation. Section 6 discusses the open issues in air pollution monitoring using UAVs. Finally, in Section 7, we present the conclusions of our work.

\section{Related Works}

UAV-based solutions have experienced a very substantial increase in the last decade, especially in the past five years. Back in 2004, NASA experts defined a wide set of civil applications for UAVs [15], highlighting their potential in the near future in areas such as commercial, Earth Sciences, national security, and land management. This preliminary report was ratified years later by authors such as Hugenholtz et al. [16], who explained how the use of UAVs could revolutionize research methods in the fields of Earth Sciences and remote sensing. In [17], authors display the results of a detailed study on different UAVs aspects, showing their applicability in Agriculture and Forestry, Disaster Monitoring, Localization and Rescue, Surveillance, Environmental Monitoring, Vegetation Monitoring, Photogrammetry, and so on.

If we focus specifically on research using quadrotor multicopters, authors like Gupte et al. [18] and Colomina and Molina [19] consider that, given their high maneuverability, compactness, and ease of use, different applications for these devices are being found in areas including civil engineering, search and rescue, emergency response, national security, military surveillance, border patrol, and surveillance, as well as in other areas such as Earth Sciences, where they can be used to study climate change, glacier dynamics, and volcanic activity or for atmospheric sampling, among others.

In our case, we are more interested in atmospheric sampling to measure air pollution levels. In this research area, Anderson and Gaston [20] highlight the applicability of UAVs in the field of ecology, emphasizing that the spatial and temporal resolutions of the data obtained by traditional methods often fail to adapt well to the requirements of local ecology-oriented research. Furthermore, the use of UAVs, when flying at low altitudes and speeds, offers new opportunities in terms of ecological phenomena measurements, enabling the delivery of data with a finer spatial resolution. In fact, Zhang and Kovacs [21] explain how the images taken by small UAVs are becoming an alternative to high-resolution satellite images, which are much more expensive, to study the variations in crop and soil conditions. Specifically, the use of UAVs is considered a good alternative given its low cost of operation in environmental monitoring, its high spatial and temporal resolution, and its high flexibility in the scheduling of image acquisitions. A good example of this use can be found in the work of Bellvert et al. [22], which shows how, by using a multicopter equipped with a thermal camera, it was possible to obtain a very precise map of water levels in a vineyard, thereby achieving significant advances in the field of precision agriculture.

Focusing on our topic, despite the presence of several works related to air pollution monitoring using Unmanned Aerial Systems (UAS), the majority of these involve, mainly, swarm creation or communication interaction between them. An example of such work is [23], where authors propose a mobility model for a group of nodes following "Virtual Tracks" (highways, valley, etc.) operating in a predefined "Switch Station" mode, through which nodes can split or merge with another group of nodes.

Different works have been done related to mounting sensors in Unmanned Aerial Vehicles. In this regard, Erman et al. [24] use an UAV equipped with a sensor to create a Wireless Sensor Network, thereby enabling each UAV to act as a sink or as a node, but it does not try to optimize the monitoring process. Teh et al. [25] propose a fixed-wind aircraft carrying a sensor node that acts as a mobile gateway, showing the communication between the UAV and different static base stations which monitor the pollution. In this case, the UAV only recovers the data collected by the stations. Khan et al. [26] propose the design of a lightweight laser-based 
sensor for measuring trace gas species using UAVs, mainly analyzing how the optical sensor captures the air pollution samples. In [27], authors use a large-sized aircraft equipped with ozone sensors to cover a wide area in an automated manner, showing how the UAV improves the sampling granularity.

If we analyze works related to mobility models for UAS mobility control that could be used for air pollution monitoring tasks, we can observe that basically no work focuses on the coverage improvement for a certain area.

For instance, in [28], authors propose a mobility model based on the Enhanced Gauss-Markov model to eliminate or limit the sudden stops and sharp turns that the random waypoint mobility model typically creates. Also, in [29], authors present a semirandom circular movement (SRCM) based model. They analyze the coverage and network connectivity by comparing results against a random waypoint mobility model.

The authors of [30] compare their models against random waypoint-based, Markov-based, and Brownian-motionbased algorithms to cover a specific area, analyzing the influence of the use of collision avoidance systems in the time to achieve full area coverage. The work in [31] compares the results of using the "Random Mobility Model" and the "Distributed Pheromone Repel Mobility Model" as direction decision engines (next waypoint) in UAV environments. The authors of [32] propose an algorithm to cover a specific area; it selects a point in space along with the line perpendicular to its heading direction and then drives the UAV based on geometric considerations.

There are works focusing on using UAVs for specific tasks involving autonomous movements. An example is [33], where authors present a mobility model for the self-deployment of an Aerial Ad Hoc Network in a disaster scenario in order to create a flying and flexible communications infrastructure that victims can use. The mobility model proposed is based mainly on the Jaccard dissimilarity metric to control the deployment of the Unmanned Aerial Vehicles composing the network. A similar work is presented in [34], where instead an in-network density analysis is used to select the physical areas that need to be visited by a flying robot.

Focusing solely on existing proposals addressing mobility models, we can find works such as [35] where authors propose the Paparazzi Mobility Model (PPRZM) by defining five types of movements-Stay-On, Waypoint, Eight, Scan, and Oval-following a defined state machine with different probabilities to change between states. There are even studies following animal-based navigation patterns. An example of such work is [36], where authors investigate the UAV placement and navigation strategies with the end goal of improving network connectivity, using local flocking rules that aerial living beings like birds and insects typically follow.

The use of UAVs for air pollution monitoring in a specific area using multirotor drones is, however, still not present in scientific literature, and this work can be seen as one of the first approaches in this direction. Our contribution can be divided into two parts: (i) the design of a low-cost and open-source UAV equipped with off-the-shelf sensors for monitoring tasks and (ii) the deployment of a protocol called

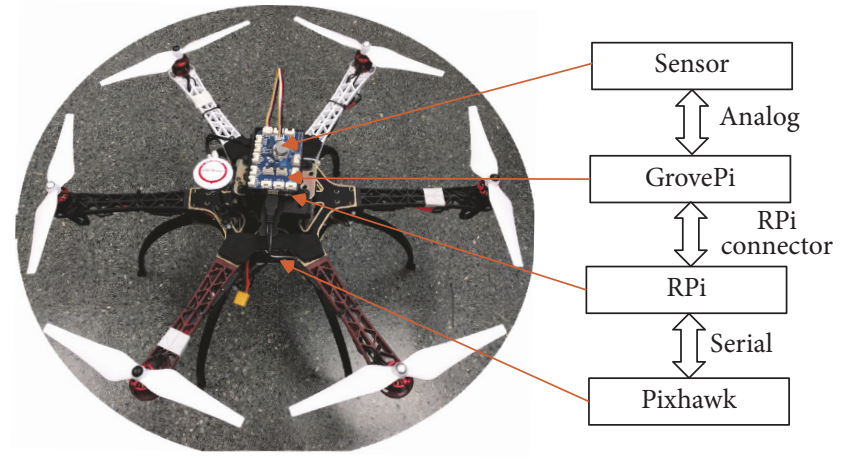

FIGURE 1: Proposed UAV with air pollution sensors.

PdUC (Pollution-driven UAV Control) to automatically track a target area by focusing on the most polluted regions.

\section{Overview of the Proposed Solution}

To implement a solution for air pollution monitoring using UAVs we have to consider, like in any cyber-physical system, two main aspects: (i) the hardware configuration and (ii) the control process for controlling the system behavior.

By following these guides, our proposal can also be split into two parts: (i) the physical configuration of the UAV and the environmental sensors and (ii) the algorithm to control the UAV for automatically monitoring a specific area called Pollution-driven UAV Control (PdUC).

Even though we are not proposing, in this paper, an implementation using real UAVs, we nevertheless present the specification of the devised cyber-physical system.

3.1. UAV Configuration. We have designed a scheme to dynamically drive the UAV by connecting the UAV control module to a Raspberry $\mathrm{Pi}$ [37] and connecting the latter to the set of pollution sensors via an analog converter. The scheme is shown in Figure 1.

The UAV is driven using a Pixhawk Autopilot $[38,39]$, which controls its physical functioning. The Raspberry $\mathrm{Pi}$ is mounted over the UAV chassis and connected to the Pixhawk through a serial port. The sensors are connected to the Raspberry Pi using a Grove Raspberry Hat (GrovePi) [40], which allows connecting different kinds of COTS sensors easily. Specifically, we are using

(i) Pixhawk Autopilot: a high-performance flight control module suitable for several types of autonomous vehicles including multirotors, helicopters, cars, boats, and fixed-wind aircrafts. It is developed under the independent, open hardware Pixhawk project, and it has two main components: (i) an Autopilot hardware provides an industry standard autopilot module designed as a $168 \mathrm{MHz}$ Cortex M4F CPU with 3D ACC/Gyro/MAG/Baro sensors, microSD slot, 5 UARTs, CAN, I2C, SPI, ADC, and so on; (ii) an Autopilot software that includes a real-time Operating System (RTOS) with a POXIS-style environment to control the drone. 


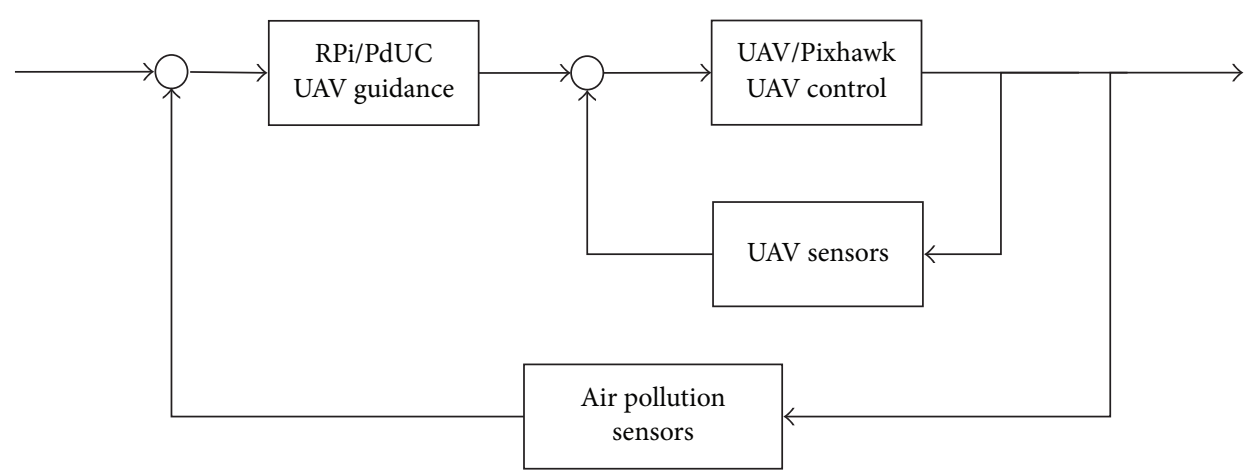

FIgURE 2: UAV control loop.

(ii) Raspberry Pi: one of the most popular single-boardcomputers (SBC) worldwide. It is a low-cost and small-sized piece of hardware that allows exploring computing and that supports different Operating Systems. The most popular of them is Raspbian, which is based on Debian, although Ubuntu Mate or Windows 10 IoT Core can also be installed, thereby allowing using several programming languages. Besides, all Raspberry Pi versions benefit from several input/output ports operating at $5 \mathrm{~V}$, thus being ideal for all sorts of IoT projects.

(iii) GrovePi: extension board that allows connecting several analog/digital grove ports to a Raspberry Pi in an easy way. It has several grove ports: seven digital ports, three analog ports, three I2C ports, one serial port to the GrovePi, and a serial connector to the Raspberry Pi.

(iv) Grove Sensors: sensors which use a grove-standardized connector, providing an easy connection to different boards like GrovePi. There are several COTS environmental sensors such as $\mathrm{CO} 2, \mathrm{CO}$, or alcohol. Specifically, we mostly focus on ozone sensors (MQ131).

Figure 2 shows the closed-loop control scheme of our proposal. The Pixhawk Autopilot is responsible for the physical control system of the UAV (lower level), while the Raspberry $\mathrm{Pi}$ is in charge of the Guidance system (higher level) determining the way forward.

3.2. Autonomous Driving. To deploy an algorithm for automatically monitoring a specific area we have analyzed, first of all, different existing possibilities that could be useful to our goals.

So, to elaborate the proposed PdUC solution, we have used specific techniques such as the metaheuristics and optimization algorithms described below.

3.2.1. Chemotaxis Metaheuristic. The use of rotary-wing UAVs, equipped with chemical sensors and tasked to survey large areas, could follow chemotactic [41] mobility patterns, since their flight behavior could easily implement the following two-phase algorithm: first, read a pollution concentration while hovering; next, follow a chemotactic step.

Chemotaxis metaheuristics are based on bacteria movement. In this model, the microorganisms react to a chemical stimulus by moving towards areas with a higher concentration of some components (e.g., food) or moving away from others (e.g., poison). In our system, we have considered the following adaptation of the chemotaxis. Let us consider an agent $i$ moving on a Euclidean plane, located at position $\vec{P}_{j}^{i}$ from an absolute reference axis, and moving along time in sequential steps $j$. For every chemotactic step, a new position $\vec{P}_{j}^{i}$ is calculated based on the previous one, defined by $x_{j-1}^{i}$ and $y_{j-1}^{i}$, plus a step size $d^{i}$ applying a random direction $\theta_{j}^{i}$, as specified in (1).

$$
\begin{gathered}
\vec{P}_{j}^{i}=\left(\begin{array}{c}
x_{j-1}^{i} \\
y_{j-1}^{i}
\end{array}\right)+\left(\begin{array}{c}
d^{i} \times \cos \left(\theta_{j}^{i}\right) \\
d^{i} \times \sin \left(\theta_{j}^{i}\right)
\end{array}\right), \\
\theta_{j}^{i}= \begin{cases}\theta_{j-1}^{i}+\alpha_{j}^{i}, & p_{j}^{i} \geq p_{j-1}^{i}, \\
-\theta_{j-1}^{i}+\beta_{j}^{i}, & p_{j}^{i}<p_{j-1}^{i} .\end{cases}
\end{gathered}
$$

The direction $\theta_{j}^{i}$, as shown in (2), is calculated on the basis of the concentration value of a certain chemical component, sampled by an agent $i$ at step $j: p_{j}^{i}$. With respect to the previously sampled value $p_{j-1}^{i}$, the following two types of movements are contemplated: Run and Tumble. In the former, Run, when the component concentration is increased with respect to the previous sample, the movement continues to follow the same direction as before $\left(\theta_{j-1}^{i}\right)$ plus a random angle $\alpha_{j}^{i}$. Regarding the latter, Tumble, when the concentration is decreasing, the movement takes a turn in the opposite direction $-\theta_{j-1}^{i}$, plus a random angle $\beta_{j}^{i}$. Notice that both $\alpha_{j}^{i}$ and $\beta_{j}^{i}$ are used to introduce variability and to maximize the gradient, allowing reaching the most polluted areas faster.

3.2.2. Particle Swarm Optimization. Particle Swarm Optimization (PSO) is a technique introduced in [42] where a solution to a problem is represented as a particle $p^{i}$ moving in a D-dimensional space at a time $t$; each particle $p^{i}$ 

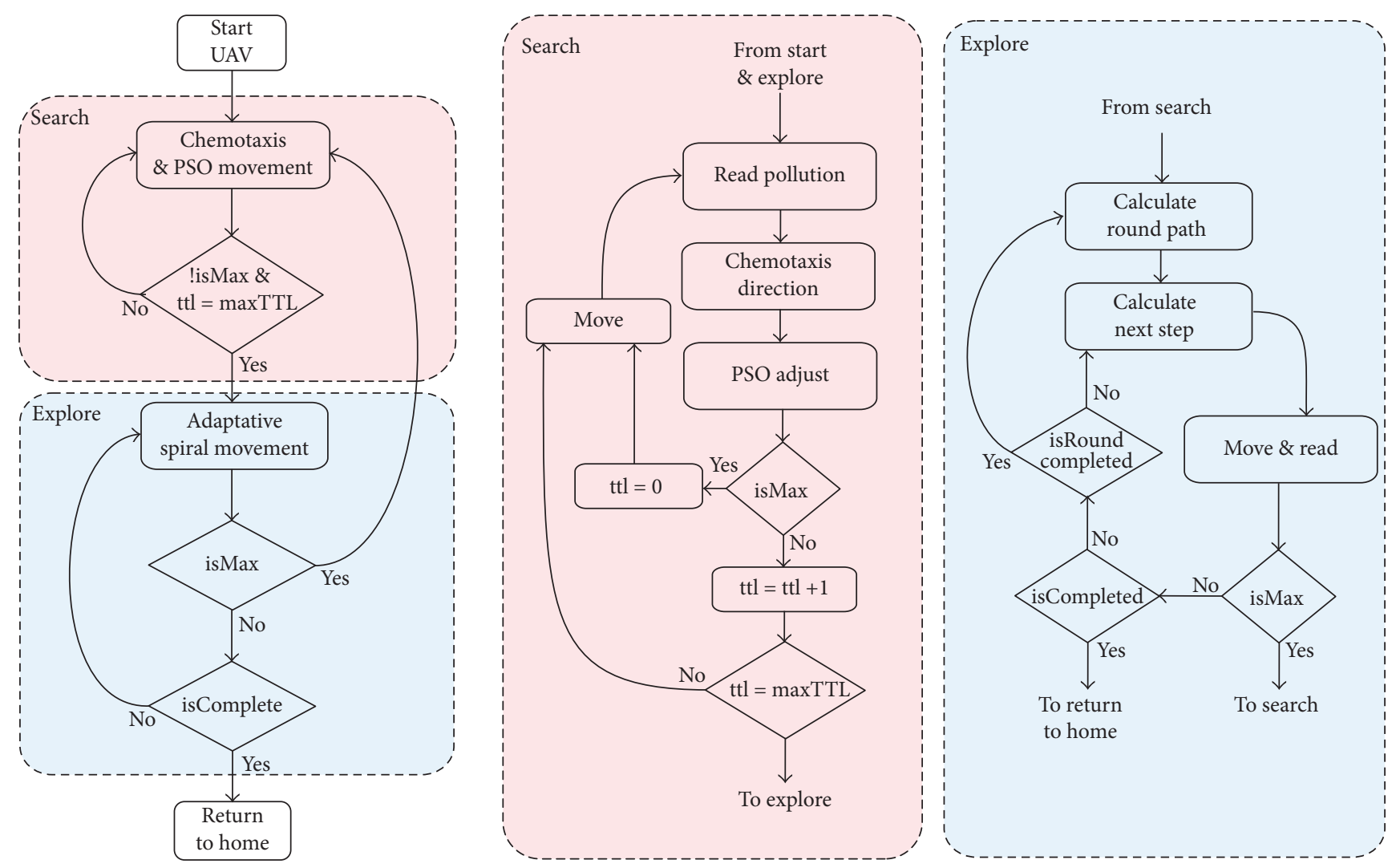

FIGURE 3: Overview of different states associated with the PdUC algorithm.

maintains its position $p_{t}^{i}$ and its best performance position $p_{b}^{i}$. To determine the next position $p_{t+1}^{i}$, PSO calculates the stochastic adjustment in the direction of the previous local best position of $i$ 's $p_{b}^{i}$ element, along with the general best position of any element $p_{b}^{g}$, as shown in

$$
\begin{aligned}
p_{t+1}^{i}= & \alpha \cdot p_{t}^{i}+U(0, \beta) \cdot\left(p_{b}^{i}-p_{t}^{i}\right)+U(0, \beta) \\
& \cdot\left(p_{b}^{g}-p_{t}^{i}\right),
\end{aligned}
$$

where $\alpha$ and $\beta$ are constants to calibrate the algorithm and $U(0, \beta)$ is a random number between $[0, \beta]$.

\section{Proposed Autonomic Solution}

To consistently drive the UAVs, so as to achieve the desired area coverage goals, we have devised the following algorithm, which incorporates a chemotactic approach.

4.1. PdUC Algorithm. In this context, we have developed an algorithm called Pollution-driven UAV Control (PdUC), based on the chemotaxis metaheuristic concept, to search an area for the highest pollution concentration levels. Once this pollution hotspot is found, the flying drone covers the whole area by following a spiral movement, starting from the most polluted location.

Our algorithm is composed of two phases: (i) a search phase, in which the UAV searches for a globally maximum pollution value, and (ii) an exploration phase, where the UAV explores the surrounding area, following a spiral movement, until one of the following conditions occurs: it covers the whole area, the allowed flight time ends, or it finds another maximum value, in which case it returns to the search phase, as shown Figure 3.

The exploration phase is based mainly on two previously described techniques: a chemotaxis metaheuristic and a local particle swarm optimization algorithm. As detailed in Algorithm 1, initially, before the UAV starts its first movement, it samples the pollution value and puts it in a buffer. For each chemotactic step, it starts to hover, collects another sample, and compares it with the previous one. If the sampling variation is positive (increasing), the UAV follows a "Run" chemotaxis direction, with a random $\alpha_{j}^{i}$ of $[-30,30]$ degrees. Otherwise, if the sampling variation is decreasing, the UAV calculates the "Tumble" chemotaxis direction in the reverse orientation with a random $\beta_{j}^{i}$ of $[-150,150]$ degrees, although modified by the actual maximum value reached $\left(m d^{i}\right)$, as shown in Figure 4. Equation (4) denotes the formula to calculate the new direction, and $\gamma$ specifies the weight of the $m d^{i}$, which must be between 0 and 1 .

To determine when PdUC has found a maximum local value, we use a TTL (time-to-live) counter. When PdUC finds a maximum value, the TTL is reset and increasing until a new maximum pollution value is found or until the maximum TTL value is reached. In this case, PdUC reverts to 


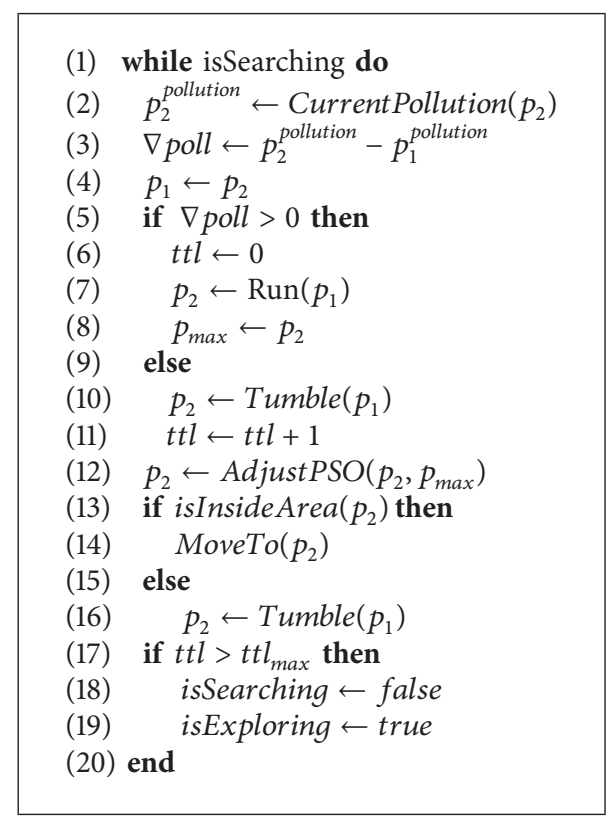

Algorithm 1: PdUC search phase.
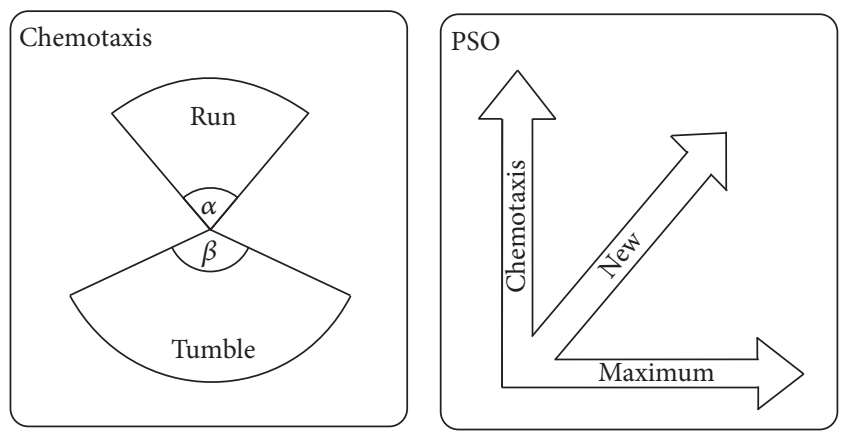

FIGURE 4: PdUC algorithm: calculation of a new direction.

the exploration phase since it considers that a local maximum value has been found.

$$
\theta_{j}^{i}= \begin{cases}\theta_{j-1}^{i}+\alpha_{j}^{i}, & \text { Run, } \\ (1-\gamma)\left(-\theta_{j-1}^{i}+\beta_{j}^{i}\right)+\gamma m d^{i}, & \text { Tumble. }\end{cases}
$$

Once a maximum value is reached, the next phase is to explore the surrounding area. As shown in algorithm 2, this is achieved by following an Archimedean spiral similar to the one depicted in Figure 5. Starting from the maximum value, it covers the surrounding area by applying a basic step size $d_{j}^{i}$ and changing it depending on the detected pollution variations, a procedure that is similar to the finding phase. If the variation is less than a preset value $c^{i}$, the step size increases until reaching $3 \times d_{j}^{i}$; otherwise, it decreases until $d_{j}^{i}$ is reached. If a maximum pollution value is found, PdUC automatically returns to the exploration phase. Finally, once

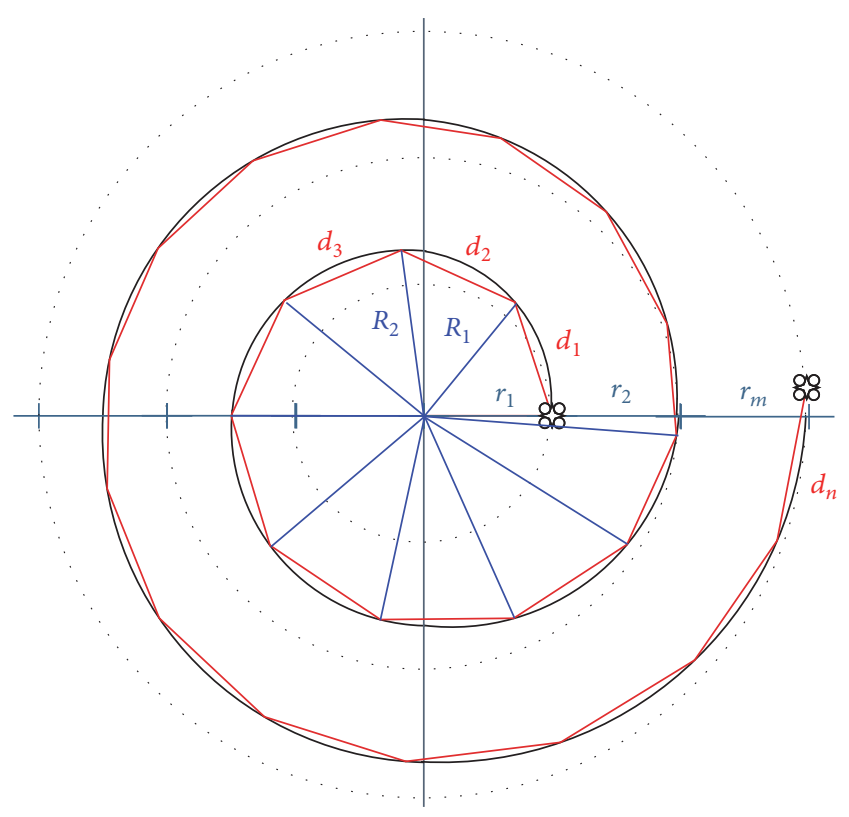

Figure 5: PdUC algorithm: exploration phase.

the whole area is covered, the UAV changes to a return-to-base (RTB) mode to finish the exploration.

4.2. Algorithm Optimization. Next, analyzing the overall behavior, we have introduced some modifications to optimize the performance of the proposed PdUC algorithm.

4.2.1. Spiralling with Alternating Directions. As shown in Figure 6, to avoid large steps in the exploration phase when the spiral center is next to a border, the direction of the spiral will alternate for each round to allow minimizing the length of some of the steps. To this purpose, for each spiral round, we calculate the direction adopted as being the opposite direction with reference to the previously used one. The system can get the general size of the area to search, as well as its borders, before starting the mission. This procedure takes place in line 4 of Algorithm 2. In detail, it follows

$$
\begin{aligned}
& \theta_{s, r}= \begin{cases}\alpha+\beta_{s, c}, & \text { if } r \text { is even, } \\
\alpha-\beta_{s, c}, & \text { if } r \text { is odd, }\end{cases} \\
& p_{s, r}=\left(\begin{array}{l}
x_{s} \\
y_{s}
\end{array}\right)=\left(\begin{array}{l}
x_{c}+R_{s} \times \cos \left(\theta_{s, r}\right) \\
y_{c}+R_{s} \times \sin \left(\theta_{s, r}\right)
\end{array}\right),
\end{aligned}
$$

where $\theta_{s, r}$ defines the angle in round $r$ and step $s, \alpha$ is the initial angle, and $\beta_{s}$ is the angle in step $s$. Using it, angle $\theta_{s, r}$ and the next point $p_{s}$ are calculated using as a reference the coordinates for the spiral center $\left(x_{c}\right.$ and $\left.y_{c}\right)$ and radius $R_{s}$.

4.2.2. Skipping Previously Monitored Areas. As shown in Figure 7, to avoid monitoring the same area multiple times, all samples, which were taken within the area monitored during the exploration phase, are internally stored. For this purpose, 


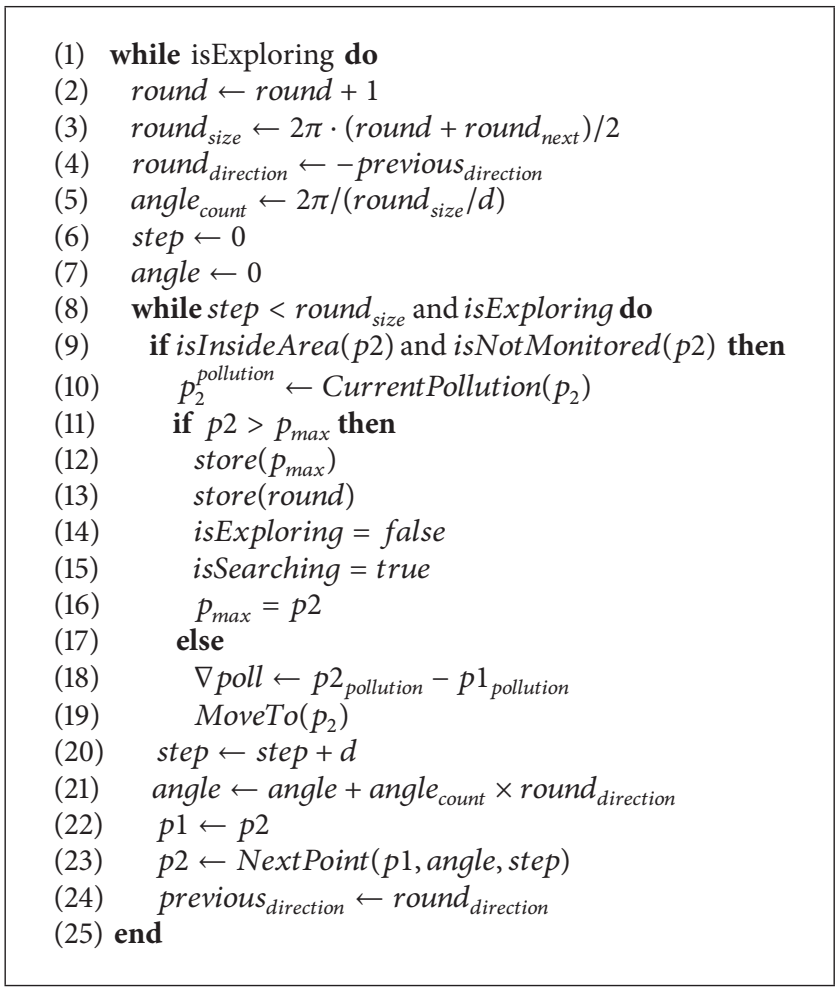

Algorithm 2: PdUC exploration phase.

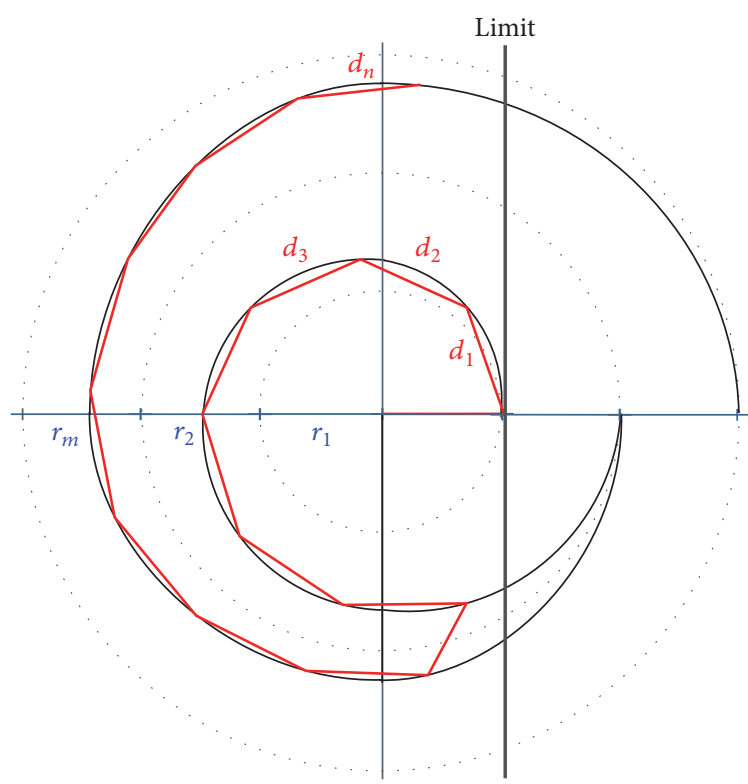

FIGURE 6: PdUC algorithm: alternating spiral direction.

PdUC maintains a list containing the location of the central position of all spirals with their respective radius to determine the monitored areas (as a circumference determined by a center and a radius). Next, in the exploration phase, all points inside these circles are omitted for the sake of celerity, as shown in line 9 of Algorithm 2.

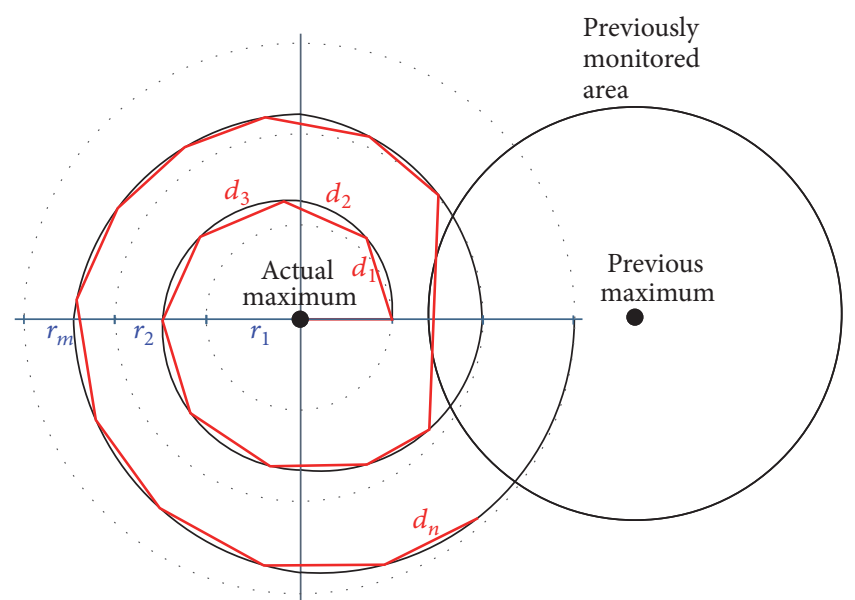

FIgURE 7: PdUC algorithm: skipping monitored areas.

\section{Validation and Simulation}

To validate our protocol, we have run several simulations with different configurations implemented in the OMNeT++ simulation tool, as shown in Figure 8.

To prepare a suitable data environment, we have created various pollution distribution maps representing ozone levels to be used as inputs for testing. These pollution maps were generated using the $R$ Graph tool [43] and following a Kriging-based interpolation [44]. In particular, a Gaussian distribution is used to adjust the parameters coming from 


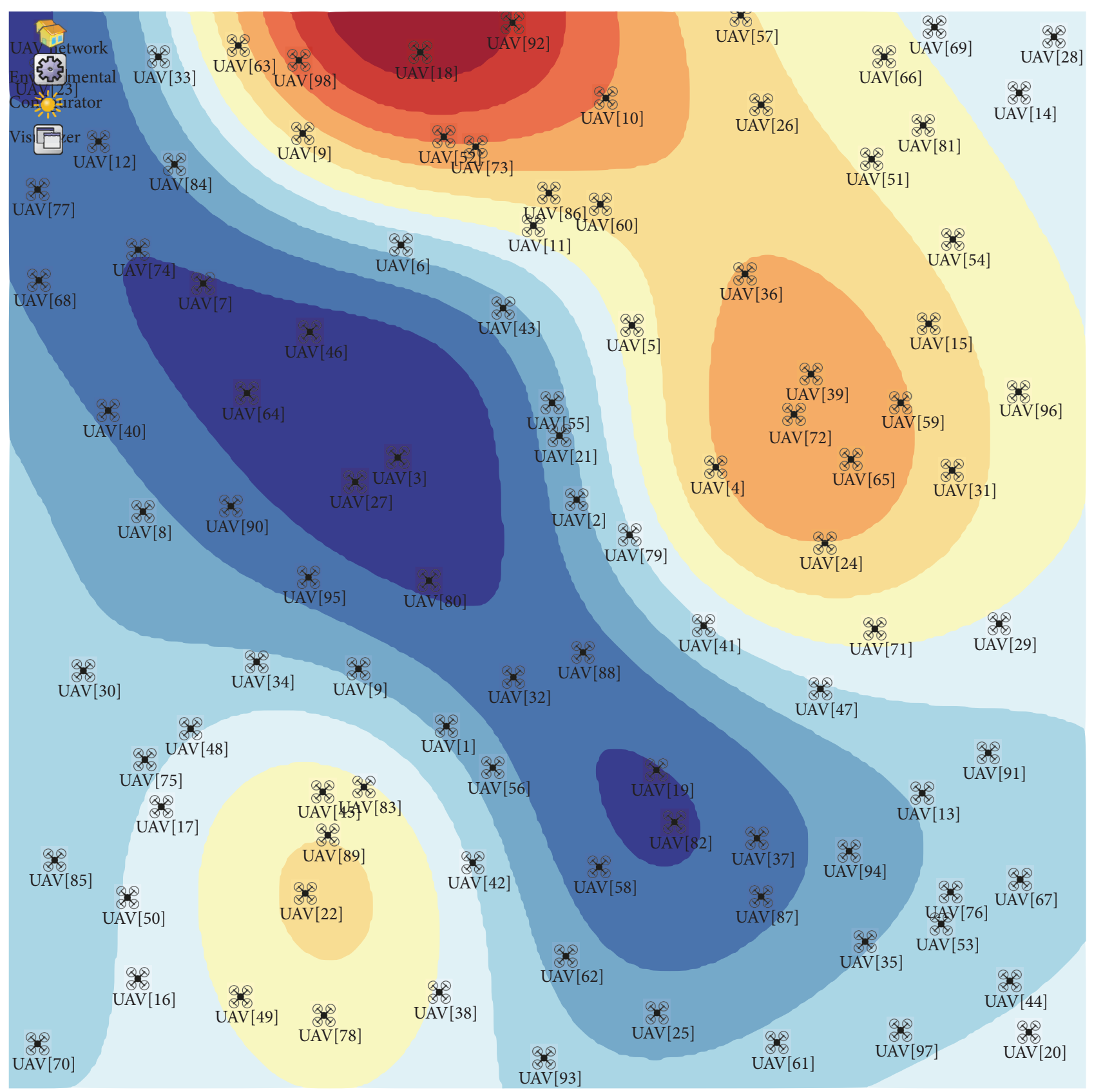

FIGURE 8: Example simulation scenario showing possible initial UAV positions over a randomly generated pollution map.

random data sources of ozone concentration. The actual values range between 40 and $180 \mathrm{ppb}$, thereby providing a realistic ozone distribution.

Figure 9 shows some samples of the created maps, which have the highest pollution concentration (areas in red) located at completely different positions due to the stochastic scenario generation procedure adopted.

Using the previously created data as input, we have run several simulations using $\mathrm{OMNeT}++$, comparing our protocol against both the Billiard and Spiral mobility patterns. In the simulator, we have created a mobility model implementation of PdUC. In addition, to simulate the sampling process, we have configured $\mathrm{OMNeT}++$ to periodically perform measurements taken from the pollution distribution map defined for the test.

Figure 10 shows an example of the path followed by an UAV using the PdUC algorithm as a guidance system. As expected, the UAV starts a search process throughout the scenario until it locates a position with the highest degree of pollution (local maximum). Afterward, it follows a spiral pattern to gain awareness of the surrounding gradients. If, while following the spiral-shaped scan path, it finds a higher 


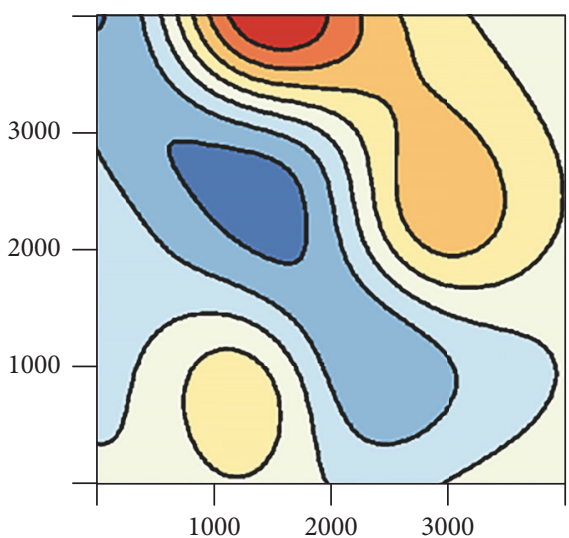

(a)

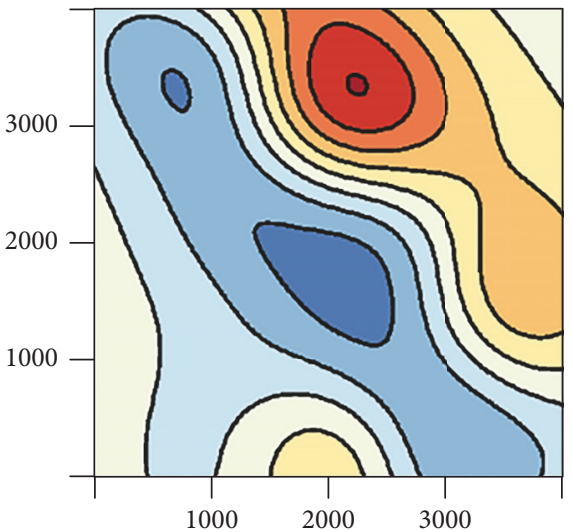

(c)
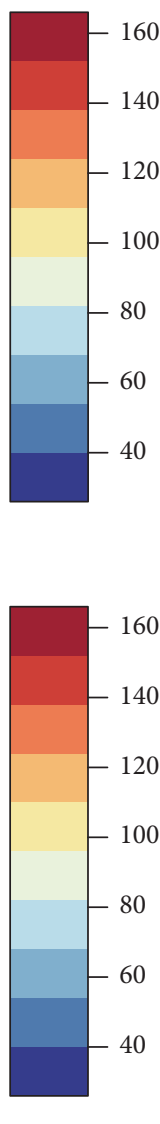

FIGURE 9: Pollution distribution examples used for validation.

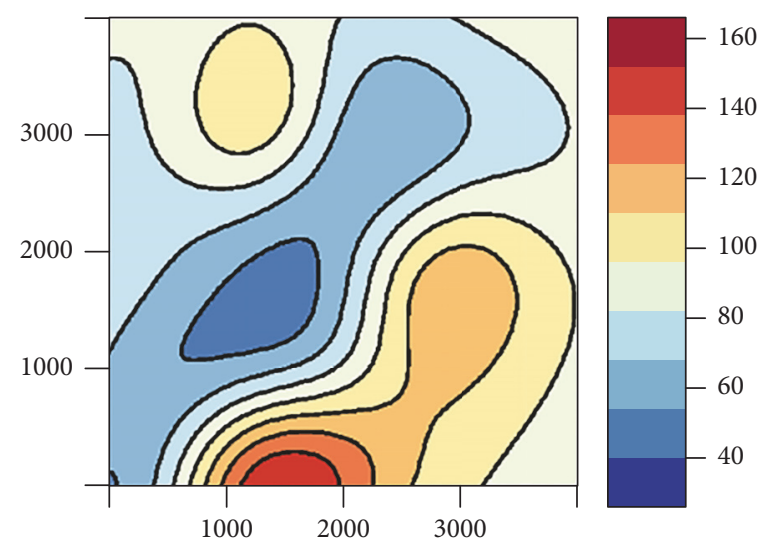

(b)

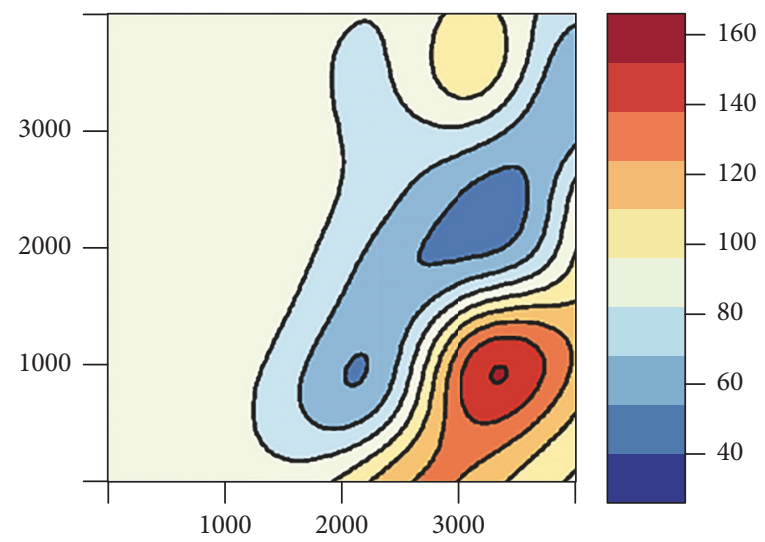

(d)

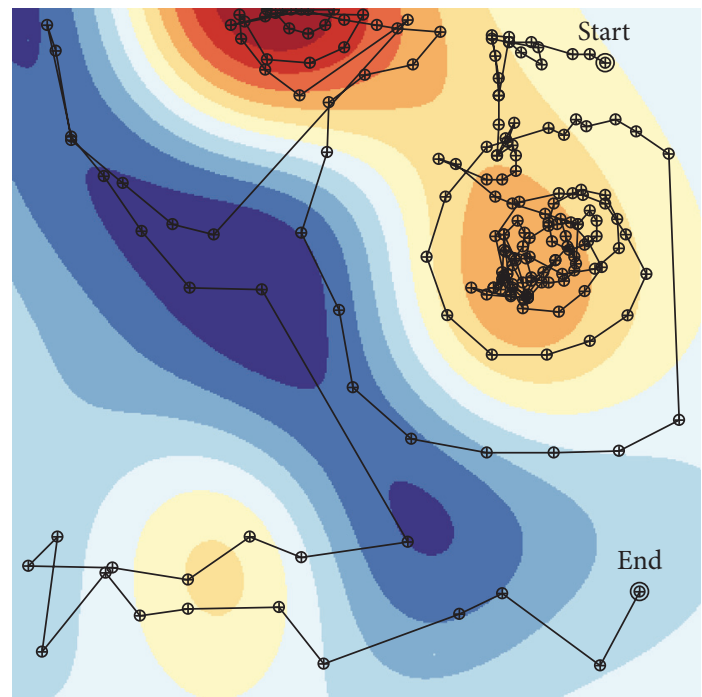

FIgUre 10: Example of an UAV path when adopting the PdUC mobility model.

pollution value, the algorithm again switches to the search phase. Finally, when the entire target area has been sampled, the algorithm finishes.
TABLE 1: Simulation parameters.

\begin{tabular}{lc}
\hline Parameter & Value \\
\hline Area & $4 \times 4 \mathrm{Km}$ \\
Pollution range & {$[40-180] \mathrm{ppb}$} \\
Sampling error & $10 \mathrm{ppb}$ \\
Max. speed & $20 \mathrm{~m} / \mathrm{s}$ \\
Sampling time & 4 seconds \\
Step distance & $100 \mathrm{~m}$ \\
Mobility models & Billiard, Spiral, and PdUC \\
\hline
\end{tabular}

To compare the three options under study, we recreate, using the R Graph tool, the pollution distribution maps using the simulation output as the input for the Kriging-based interpolation. In this way, we obtain new pollution maps for comparison against the ones used as reference.

Table 1 summarizes the parameters used in the simulations.

Since we are proposing the PdUC algorithm for rural environments, the simulation area defined is a $4 \times 4 \mathrm{Km}$ area. As indicated above, the pollution distribution relies on synthetic maps that are generated by combining a random Kriging interpolation following a Gaussian model with values between 40 and 180 units based on the Air Quality Index 


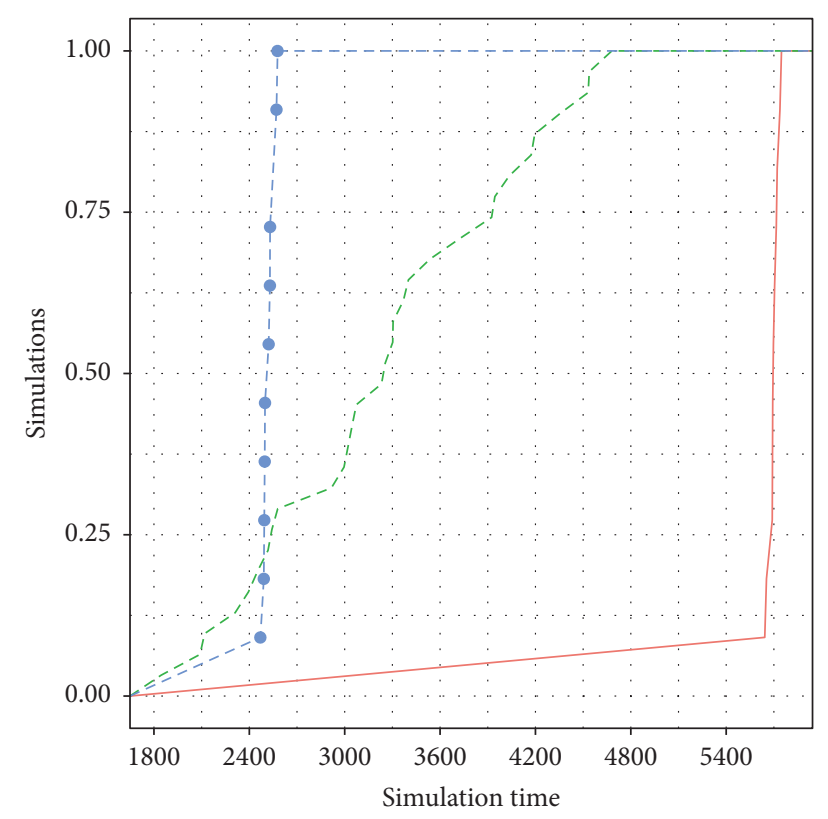

Mobility model
- Billiard
-- PdUC
-- Spiral

FIGURE 11: Cumulative Distribution Function of the time spent at covering the complete area for the Billiard, Spiral, and PdUC mobility models.

(AQI) [45]. Since samples are taken using off-the-shelf sensors, which are not precise, we introduce a random sampling error of $\pm 10 \mathrm{ppb}$ based on real tests using the MQ131 (Ozone) sensor. In our simulation, we set the maximum UAV speed to $20 \mathrm{~m} / \mathrm{s}$, a value achievable by many commercial UAVs. The step distance defined between consecutive samples is 100 meters. Once a new sampling location is reached, the monitoring time per sample is defined to be 4 seconds.

The mobility models used are Billiard, Spiral, and PdUC. These models have different assumptions regarding the initial UAV position. In the Billiard model, the UAV starts in a corner of the target area and then covers the whole area by "bouncing" when reaching the borders. The Spiral model starts at the center of the area to cover and then gradually moves to the periphery of the scenario following a spiral pattern. Finally, PdUC is set to start at a random position within the target area.

We now proceed by analyzing the time required to cover the entire area using each of the approaches being tested. For this purpose, we defined 100 simulations for each model (Billiard, Spiral, and PdUC) and determined the required time to cover the whole area, estimating the pollution map afterward.

For each run, the starting position of the UAV is randomly set on the map, as shown in Figure 8.

Figure 11 shows the Cumulative Distribution Function relative to the time required to cover the whole area for the three mobility models. It can be seen that the Billiard and

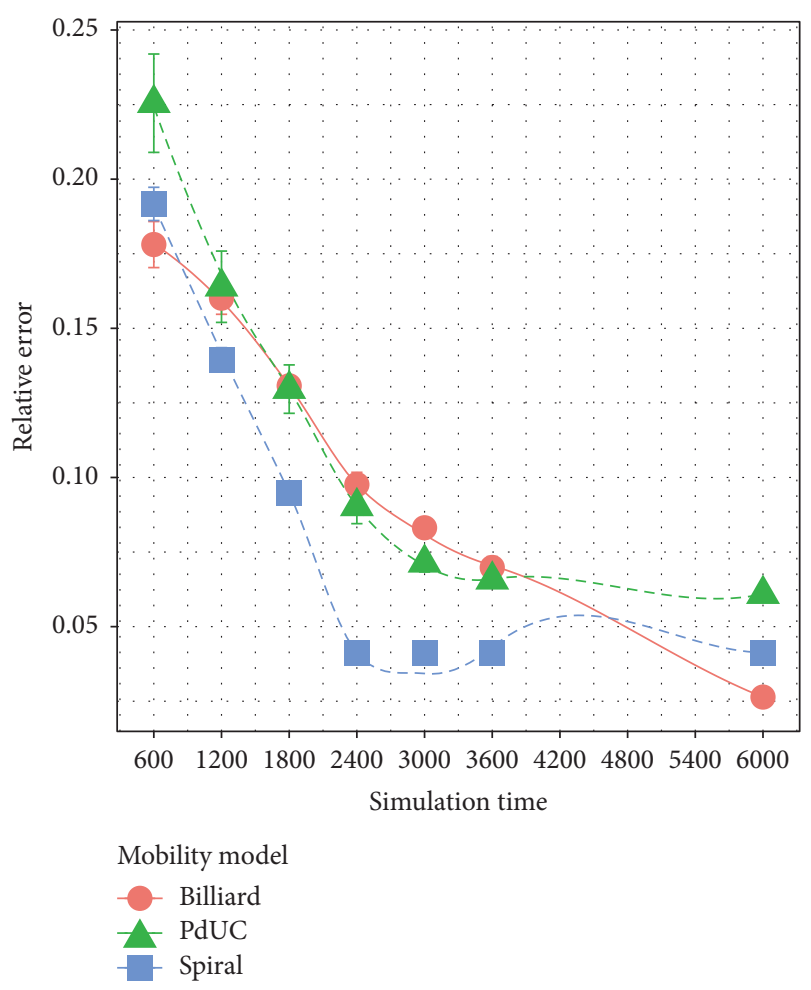

FIGURE 12: Relative error comparison between the PdUC, Billiard, and Spiral mobility models at different times, when analyzing all the values.

Spiral models do not depend on the start position, spending a nearly constant time (5600 and 2600 seconds, resp.) for each configuration defined. In the case of the PdUC mobility model, since it reacts to air pollution, the time required to cover the complete area varies between 1800 and 4300 seconds, depending on the start position.

Due to battery restrictions, it is interesting to analyze how fast each mobility model discovers the most polluted areas and how accurately does it recreate the pollution distribution. For this purpose, we analyze the relative error for the three mobility models at different time instants $(600,1200,1800$, 2400,3000 , and 6000 seconds); this error is defined by

$$
e_{t}=\frac{\sum_{i=1}^{m} \sum_{j=1}^{n}\left|\left(s_{x, y, t}-b_{x, y}\right) / \Delta b\right|}{m \cdot n}
$$

where $e_{t}$ is the relative error at time $t ; s_{x, y, t}$ is the recreated pollution value at position $(x, y)$ using the samples taken during simulation until time $t, b_{x, y}$ is the reference pollution value at position $(x, y)$, and $n$ and $m$ are the dimensions of the target area, respectively.

Figure 12 shows the temporal evolution of the relative error between the three mobility models (Billiard, Spiral, and PdUC) and the original one. We can observe that all mobility models have roughly the same behavior: they start with a high relative error, which is foreseeable since we are using Kriging interpolation to recreate the pollution distribution, and it tends to the mean value when the number of samples 


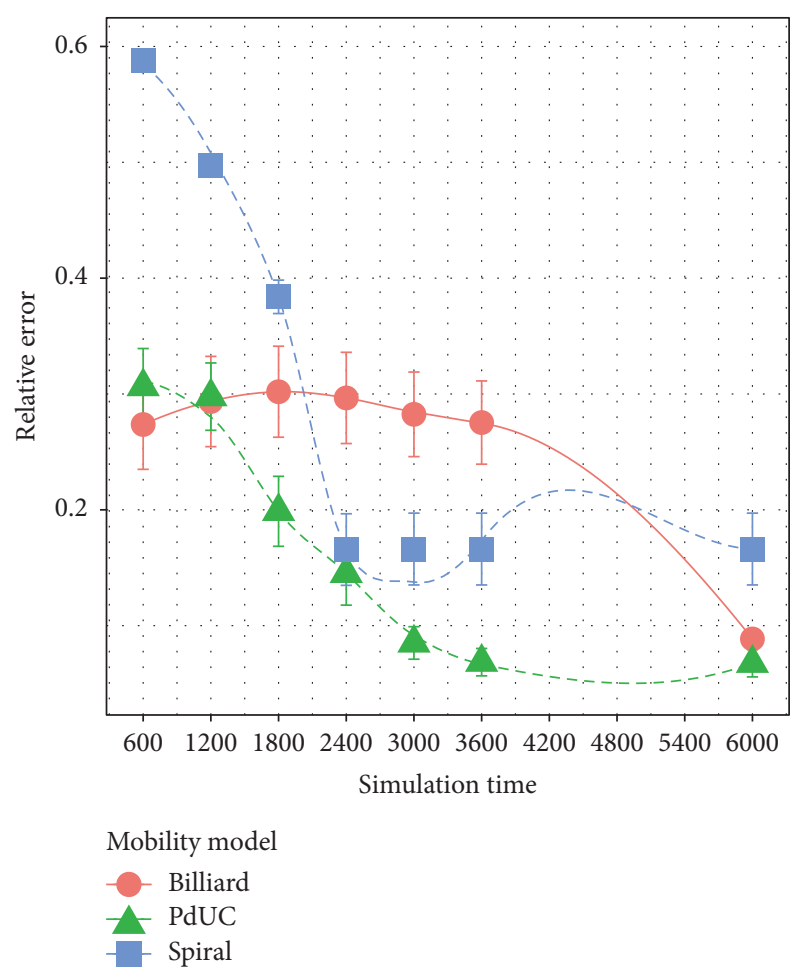

FIGURE 13: Relative error comparison between PdUC, Billiard, and Spiral mobility models at different times when only considering values higher than $120 \mathrm{ppb}$.

is not enough. Then, as more samples become available, the spatial interpolation process quickly becomes more precise.

Although the three mobility models are similar, the spiral approach achieves a better performance in terms of relative error reduction. However, if we analyze only the most polluted regions, that is, regions characterized by values higher than a certain threshold (120 and $150 \mathrm{ppm}$ in our case, based on AQI [45]), we find that PdUC clearly provides better results.

Figures 13 and 14 show the comparison between the Billiard, Spiral, and PdUC mobility models at different times when only focusing on air pollution values higher than 120 and $150 \mathrm{ppb}$, respectively. These results show that PdUC clearly provides better results than the Billiard and Spiral movement patterns, outperforming their accuracy from nearly the beginning of the experiment (1200 seconds) and reaching the lowest relative error values in just 3600 seconds, with these two other mobility approaches more than doubling the error values for the same time. In particular, the Billiard mobility pattern requires about 6000 seconds to achieve a similar degree of accuracy (120 ppb case), while the Spiral approach is not able to achieve values as low as PdUC in any of the cases. This occurs because PdUC focuses on the highest values in the chemotaxis-based phase. PdUC always prioritizes the most polluted areas in detriment of less polluted ones, thus allowing obtaining, at least, details about the region with the highest pollution values.

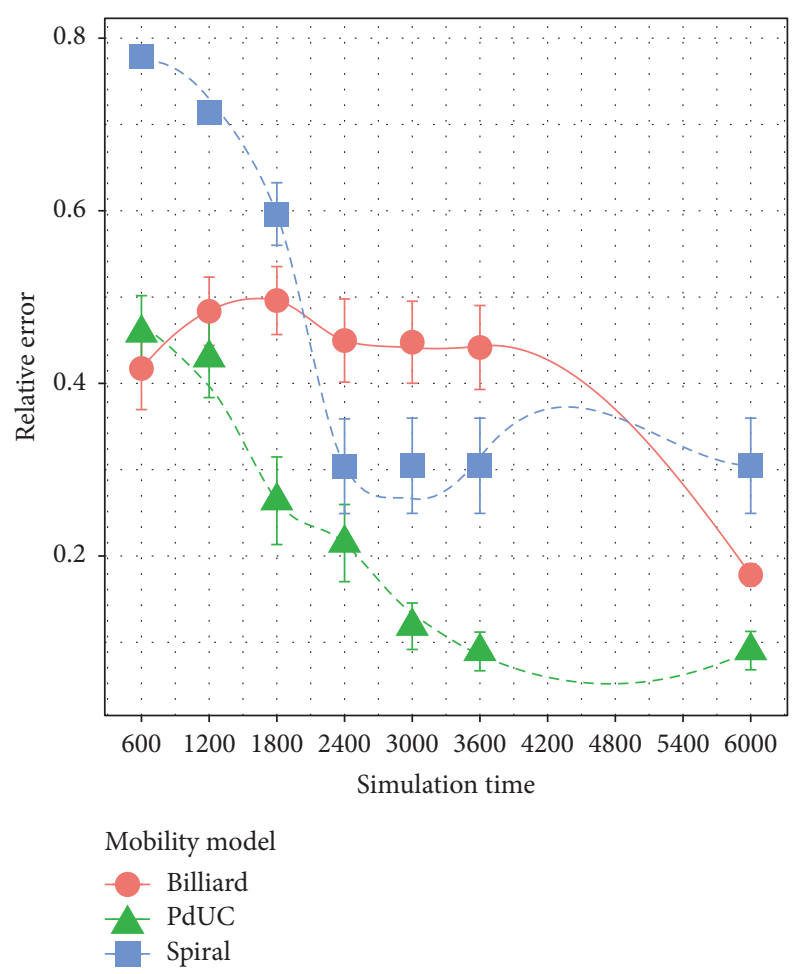

Figure 14: Relative error comparison between PdUC, Billiard, and Spiral mobility models at different times when only considering values higher than $150 \mathrm{ppb}$.

To complete our study, Figure 15 presents an example of the evolution of predicted pollution values for the whole target area and for the three algorithms under analysis (Spiral, Billiard, and PdUC), at different times (1200 s, $2400 \mathrm{~s}, 3600 \mathrm{~s}$, and $6000 \mathrm{~s}$ ). We can observe that PdUC is able to quickly find the most polluted areas, while the effectiveness of other approaches highly depends on the actual location of pollution hotspots in order to detect them at an early stage.

\section{Open Issues}

Unmanned Aerial Systems (UAS) have been quickly adopted in different application areas due to their flexibility and relatively low cost. Focusing on the environmental monitoring area, in a previous work we introduced the idea of using UAVs for air pollution monitoring [46] by equipping them with offthe-shelf sensors. Instead, in the current paper, we introduce an algorithm called PdUC to guide a single UAV in the task of monitoring a specific area. However, there are still several open issues related to this topic.

Until now, we have only considered operations limited to a single UAV. The next step in our research is to introduce multiple-UAVs and the associated cooperation schemes. The following aspects need to be addressed to follow this research line:

(i) Cooperation. To maximize the effectiveness and reduce mapping times, it is advisable to have several 


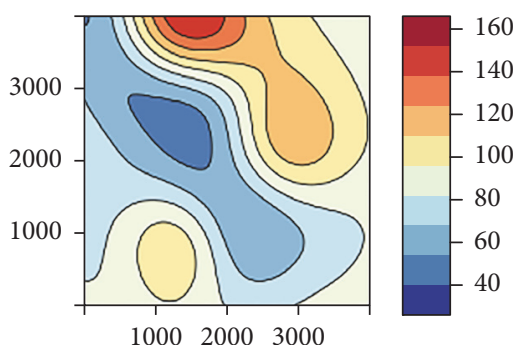

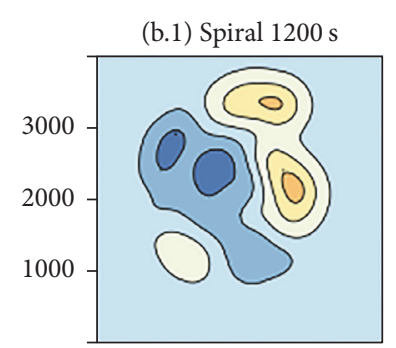

(b.2) Spiral $2400 \mathrm{~s}$

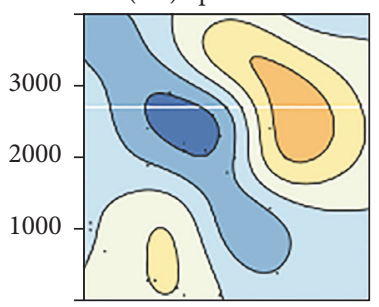

(b.3) Spiral $3600 \mathrm{~s}$

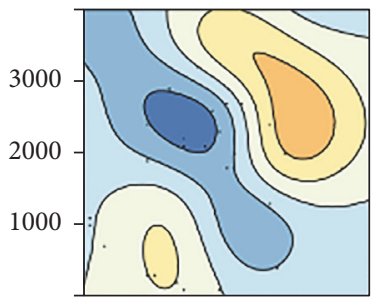

(b.4) Spiral $6000 \mathrm{~s}$

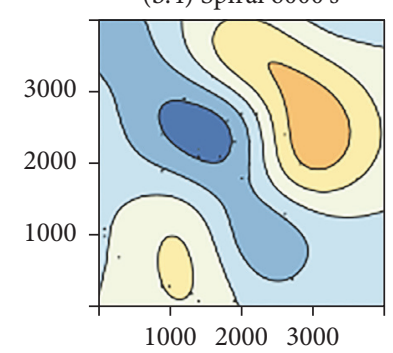

(c.1) Billiard $1200 \mathrm{~s}$

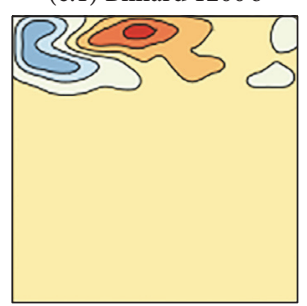

(c.2) Billiard $2400 \mathrm{~s}$

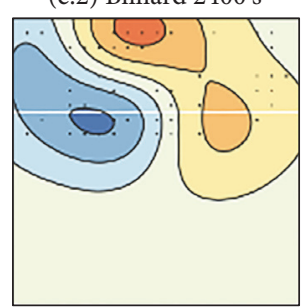

(c.3) Billiard $3600 \mathrm{~s}$

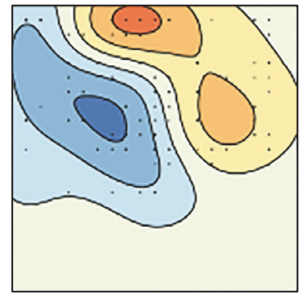

(c.4) Billiard $6000 \mathrm{~s}$

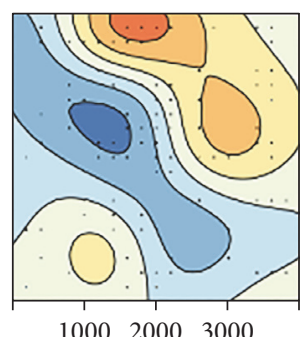

(d.1) PdUC $1200 \mathrm{~s}$

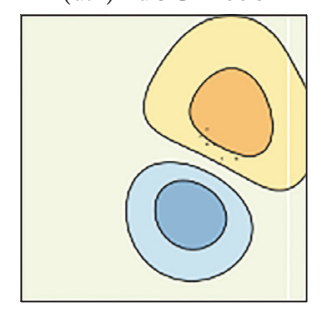

(d.2) PdUC $2400 \mathrm{~s}$

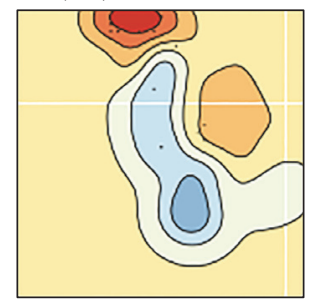

(d.3) PdUC $3600 \mathrm{~s}$

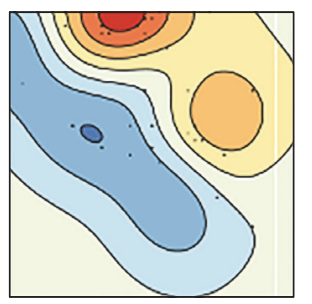

(d.4) PdUC 6000 s

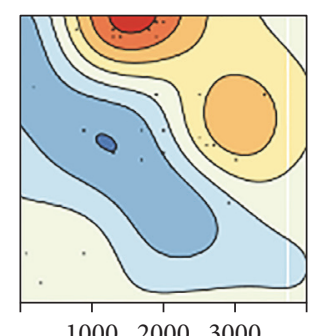

160

140

120

$-80$

$-60$

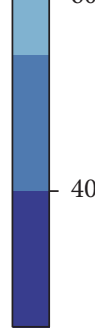

FIGURE 15: Visual representation of the estimation output for the PdUC, Billiard, and Spiral mobility models at different times.

UAVs that cooperate with each other to achieve the same task, thereby accelerating the whole process and avoiding battery exhaustion before completing the monitoring process.

(ii) Collision Avoidance. Since the different UAVs are expected to have some degree of autonomy regarding their mobility pattern, a correct coordination between nearby UAVs is required to avoid collisions when flying at a close range. (iii) Communications. To achieve the aforementioned goals of cooperation and collision avoidance, communications between UAVs and between UAVs and a central management unit are required.

On the other hand, using mobile sensors installed on UAVs introduces new issues to the sensing process that should also be addressed:

(i) Altitude. Despite the fact that currently most pollution studies are made at a ground level, the use 
of UAVs allows determining the concentration of pollutants at different heights, allowing determining if there are layers of pollutants that can cause health problems in rugged mountainsides.

(ii) Influence of the Wind. The sampling procedure includes sensors that are sensitive to the wind conditions. In addition, wind causes the overall pollution map to be more dynamic. In this context, both issues deserve more scrutiny.

\section{Conclusions}

Despite the fact that we have several options to monitor air pollution in urban scenarios, being crowdsensing an emerging approach arousing great interest, finding an adequate approach for industrial or rural areas remains a pending task.

Recently, Unmanned Aerial Systems have experienced unprecedented growth, offering a platform for the fast development of solutions due to their flexibility and relatively low cost; in fact, they can be good options to solve the previous requirements, allowing monitoring remote areas that are difficult to access.

In this paper, we propose a solution where we equip an UAV with off-the-shelf sensors for monitoring tasks, using a Pixhawk Autopilot for UAV control, and a Raspberry Pi for sensing and storing environmental pollution data.

To automatically analyze pollution values within a target area, we also propose an adaptive algorithm for autonomous navigation called Pollution-based UAV Control system (PdUC). This algorithm allows an UAV to autonomously monitor a specific area by prioritizing the most polluted zones. In particular, PdUC combines different concepts including a chemotaxis metaheuristic, a local particle swarm optimization (PSO), and an Adaptive Spiralling technique, to create an algorithm able to quickly search for hotspots having high pollution values, and to cover the surrounding area as well, thereby obtaining a complete and detailed pollution map of the target region.

To validate our proposal, we compared the proposed PdUC solution against the Billiard and Spiral mobility models through simulations implemented in $\mathrm{OMNeT}++$. Simulation experiments show that PdUC offers significantly better performance at reducing prediction errors, especially regarding the accuracy achieved for the high-values range.

\section{Conflicts of Interest}

The received funding does not lead to any conflicts of interest regarding the publication of the manuscript.

\section{Acknowledgments}

This work has been partially carried out in the framework of the DIVINA Challenge Team, which is funded under the Labex MS2T program. Labex MS2T is supported by the French Government, through the program "Investments for the Future" managed by the National Agency for Research (Reference: ANR-11-IDEX-0004-02). This work was also supported by the "Programa Estatal de Investigación, Desarrollo e Innovación Orientada a Retos de la Sociedad, Proyecto I+D+I TEC2014-52690-R," the "Programa de Becas SENESCYT de la República del Ecuador," and the Research Direction of University of Cuenca.

\section{References}

[1] A. Seaton, D. Godden, W. MacNee, and K. Donaldson, "Particulate air pollution and acute health effects," The Lancet, vol. 345, no. 8943 , pp. $176-178,1995$.

[2] Q. S. McFrederick, J. C. Kathilankal, and J. D. Fuentes, "Air pollution modifies floral scent trails," Atmospheric Environment, vol. 42, no. 10, pp. 2336-2348, 2008.

[3] D. Mage, G. Ozolins, P. Peterson et al., "Urban air pollution in megacities of the world," Atmospheric Environment, vol. 30, no. 5, pp. 681-686, 1996.

[4] H. Mayer, "Air pollution in cities," Atmospheric Environment, vol. 33, no. 24-25, pp. 4029-4037, 1999.

[5] P. S. Kanaroglou, M. Jerrett, J. Morrison et al., "Establishing an air pollution monitoring network for intra-urban population exposure assessment: A location-allocation approach," Atmospheric Environment, vol. 39, no. 13, pp. 2399-2409, 2005.

[6] O. Alvear, W. Zamora, C. Calafate, J.-C. Cano, and P. Manzoni, "An architecture offering mobile pollution sensing with high spatial resolution," Journal of Sensors, vol. 2016, Article ID 1458147, 2016.

[7] A. Adam-Poupart, A. Brand, M. Fournier, M. Jerrett, and A. Smargiassi, "Spatiotemporal modeling of ozone levels in Quebec (Canada): a comparison of kriging, land-use regression (LUR), and combined bayesian maximum entropy-LUR approaches," Environmental Health Perspectives, vol. 122, no. 9, pp. 970-976, 2014.

[8] M. Pujadas, J. Plaza, J. Terés, B. Artíñano, and M. Millán, "Passive remote sensing of nitrogen dioxide as a tool for tracking air pollution in urban areas: The Madrid urban plume, a case of study," Atmospheric Environment, vol. 34, no. 19, pp. 3041-3056, 2000.

[9] S. B. Eisenman, E. Miluzzo, N. D. Lane, R. A. Peterson, G.-S. Ahn, and A. T. Campbell, "BikeNet: a mobile sensing system for cyclist experience mapping," ACM Transactions on Sensor Networks, vol. 6, pp. 87-101, 2009.

[10] M. André, "The ARTEMIS European driving cycles for measuring car pollutant emissions," Science of the Total Environment, vol. 334-335, pp. 73-84, 2004.

[11] M. Brkovic and V. Sretovic, "Urban sensingsmart solutions for monitoring environmental quality: Case studies from serbia in. 48th ISOCARP International Congress: Fast Forward: Planning in a (hyper) dynamic urban context," Perm, Russia, 2012.

[12] S.-C. Hu, Y.-C. Wang, C.-Y. Huang, and Y.-C. Tseng, "Measuring air quality in city areas by vehicular wireless sensor networks," Journal of Systems and Software, vol. 84, no. 11, pp. 2005-2012, 2011.

[13] Y. Cheng, X. Li, Z. Li et al., "AirCloud: a cloud-based air-quality monitoring system for everyone," in Proceedings of the 12th ACM Conference on Embedded Network Sensor Systems (SenSys '14), pp. 251-265, Memphis, TN, USA, November 2014.

[14] M. Dunbabin and L. Marques, "Robots for environmental monitoring: Significant advancements and applications," IEEE Robotics and Automation Magazine, vol. 19, no. 1, pp. 24-39, 2012. 
[15] T. H. Cox, C. J. Nagy, M. A. Skoog, I. A. Somers, and R. Warner, Civil uav capability assessment.

[16] C. H. Hugenholtz, B. J. Moorman, K. Riddell, and K. Whitehead, "Small unmanned aircraft systems for remote sensing and Earth science research," Eos, Transactions American Geophysical Union, vol. 93, no. 25, p. 236, 2012.

[17] G. Pajares, "Overview and current status of remote sensing applications based on unmanned aerial vehicles (UAVs)," Photogrammetric Engineering and Remote Sensing, vol. 81, no. 4, pp. 281-329, 2015.

[18] S. Gupte, P. I. T. Mohandas, and J. M. Conrad, "A survey of quadrotor unmanned aerial vehicles," in Proceedings of the Proceeding of the IEEE Southeastcon, pp. 1-6, Orlando, Fla, USA, March 2012.

[19] I. Colomina and P. Molina, "Unmanned aerial systems for photogrammetry and remote sensing: a review," ISPRS Journal of Photogrammetry and Remote Sensing, vol. 92, pp. 79-97, 2014.

[20] K. Anderson and K. J. Gaston, "Lightweight unmanned aerial vehicles will revolutionize spatial ecology," Frontiers in Ecology and the Environment, vol. 11, no. 3, pp. 138-146, 2013.

[21] C. Zhang and J. M. Kovacs, "The application of small unmanned aerial systems for precision agriculture: a review," Precision Agriculture, vol. 13, no. 6, pp. 693-712, 2012.

[22] J. Bellvert, P. J. Zarco-Tejada, J. Girona, and E. Fereres, "Mapping crop water stress index in a 'Pinot-noir' vineyard: Comparing ground measurements with thermal remote sensing imagery from an unmanned aerial vehicle," Precision Agriculture, vol. 15, no. 4, pp. 361-376, 2014.

[23] B. Zhou, K. Xu, and M. Gerla, "Group and swarm mobility models for ad hoc network scenarios using virtual tracks," in Proceedings of the OtLCOM 2004 - 2004 IEEE Military Communications Conference, pp. 289-294, November 2004.

[24] A. T. Erman, L. V. Hoesel, P. Havinga, and J. Wu, "Enabling mobility in heterogeneous wireless sensor networks cooperating with UAVs for mission-critical management," IEEE Wireless Communications, vol. 15, no. 6, pp. 38-46, 2008.

[25] S. K. Teh, L. Mejias, P. Corke, and W. Hu, "Experiments in integrating autonomous uninhabited aerial vehicles(uavs) and wireless sensor networks," in Proceedings of the Australasian Conference on Robotics and Automation (ACRA 08, The Australian Robotics and Automation Association Inc), Canberra, Australia, 2008, https://eprints.qut.edu.au/15536/.

[26] A. Khan, D. Schaefer, L. Tao et al., "Low power greenhouse gas sensors for unmanned aerial vehicles," Remote Sensing, vol. 4, no. 5, pp. 1355-1368, 2012.

[27] S. Illingworth, G. Allen, C. Percival et al., "Measurement of boundary layer ozone concentrations on-board a Skywalker unmanned aerial vehicle," Atmospheric Science Letters, vol. 15, no. 4, pp. 252-258, 2014.

[28] J.-D. M. M. Biomo, T. Kunz, and M. St-Hilaire, "An enhanced Gauss-Markov mobility model for simulations of unmanned aerial ad hoc networks," in Proceedings of the 7th IFIP Wireless and Mobile Networking Conference, WMNC 2014, Vilamoura, Portugal, May 2014.

[29] W. Wang, X. Guan, B. Wang, and Y. Wang, "A novel mobility model based on semi-random circular movement in mobile ad hoc networks," Information Sciences, vol. 180, no. 3, pp. 399-413, 2010.

[30] D. Orfanus and E. P. De Freitas, "Comparison of UAV-based reconnaissance systems performance using realistic mobility models," in Proceedings of the 2014 6th International Congress on Ultra Modern Telecommunications and Control Systems and Workshops, ICUMT 2014, pp. 248-253, Saint Petersburg, Russia, October 2014.

[31] E. Kuiper and S. Nadjm-Tehrani, "Mobility models for UAV group reconnaissance applications," in Proceedings of the 2nd International Conference on Wireless and Mobile Communications (ICWMC '06), pp. 33-43, July 2006.

[32] Y. Wan, K. Namuduri, Y. Zhou, and S. Fu, "A smooth-turn mobility model for airborne networks," IEEE Transactions on Vehicular Technology, vol. 62, no. 7, pp. 3359-3370, 2013.

[33] J. Sanchez-Garcia, J. M. Garcia-Campos, S. L. Toral, D. G. Reina, and F. Barrero, "A Self Organising Aerial Ad Hoc Network Mobility Model for Disaster Scenarios," in Proceedings of the 8th International Conference on Developments in eSystems Engineering, DeSE 2015, pp. 35-40, December 2015.

[34] O. Briante, V. Loscri, P. Pace, G. Ruggeri, and N. R. Zema, "COMVIVOR: An Evolutionary Communication Framework Based on Survivors' Devices Reuse," Wireless Personal Communications, vol. 85, no. 4, pp. 2021-2040, 2015.

[35] O. Bouachir, A. Abrassart, F. Garcia, and N. Larrieu, "A mobility model for UAV ad hoc network," in Proceedings of the 2014 International Conference on Unmanned Aircraft Systems, ICUAS 2014, pp. 383-388, Orlando, FL, USA, May 2014.

[36] P. Basu, J. Redi, and V. Shurbanov, "Coordinated flocking of UAVS for improved connectivity of mobile ground nodes," in Proceedings of the MILCOM 2004 - 2004 IEEE Military Communications Conference, pp. 1628-1634, November 2004.

[37] R. P. Foundation, "Raspberry pi," https://www.raspberrypi.org/.

[38] L. Meier, P. Tanskanen, F. Fraundorfer, and M. Pollefeys, "PIXHAWK: a system for autonomous flight using onboard computer vision," in Proceedings of the IEEE International Conference on Robotics and Automation (ICRA '11), pp. 2992-2997, IEEE, Shanghai, China, May 2011.

[39] L. Meier, P. Tanskanen, L. Heng, G. H. Lee, F. Fraundorfer, and M. Pollefeys, "PIXHAWK: a micro aerial vehicle design for autonomous flight using onboard computer vision," Autonomous Robots, vol. 33, no. 1-2, pp. 21-39, 2012.

[40] D. Industries, Grovepi, http://www.dexterindustries.com/ grovepi/.

[41] I. BoussaïD, J. Lepagnot, and P. Siarry, "A survey on optimization metaheuristics," Information Sciences. An International Journal, vol. 237, pp. 82-117, 2013.

[42] R. C. Eberhart and J. Kennedy, "A new optimizer using particle swarm theory," in Proceedings of the 6th International Symposium on Micromachine and Human Science, pp. 39-43, Nagoya, Japan, October 1995.

[43] R. Core Team, "R: A Language and Environment for Statistical Computing, R Foundation for Statistical Computing, Vienna, Austria," 2016, http://www.R-project.org/.

[44] M. L. Stein, Interpolation of Spatial Data: Some Theory for Kriging, Springer, New York, NY, USA, 1999.

[45] U. S. E. P. Agency, "Air Quality Index," 2015, http://cfpub.epa .gov/airnow/index.cfm?action=aqibasics.aqi.

[46] O. Alvear, C. T. Calafate, E. Hernández, J.-C. Cano, and P. Manzoni, "Mobile Pollution Data Sensing Using UAVs," in Proceedings of the 13th International Conference on Advances in Mobile Computing and Multimedia, MoMM 2015, pp. 393-397, Brussels, Belgium, December 2015. 


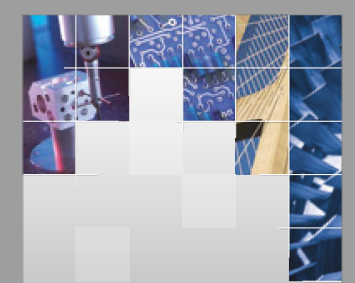

\section{Enfincering}
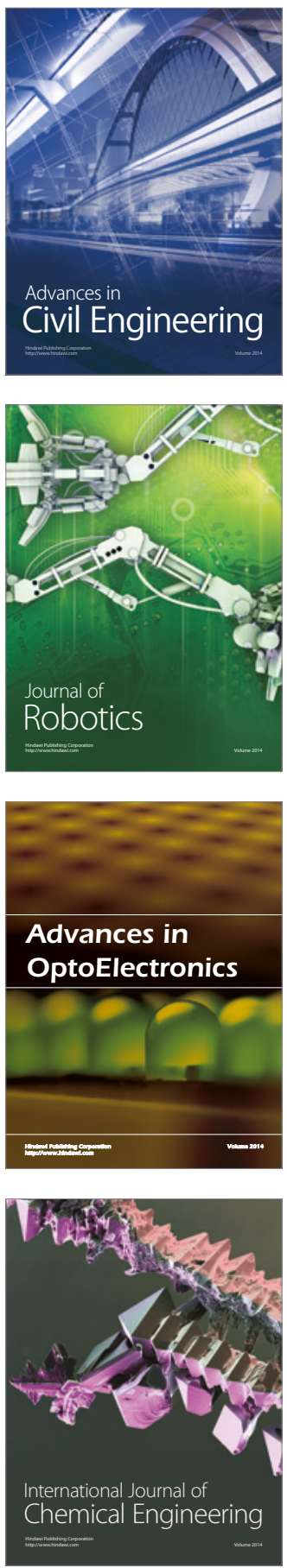

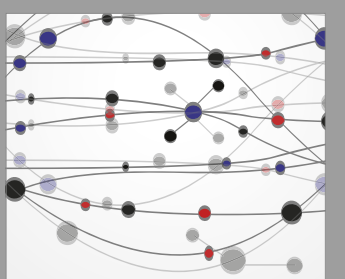

The Scientific World Journal

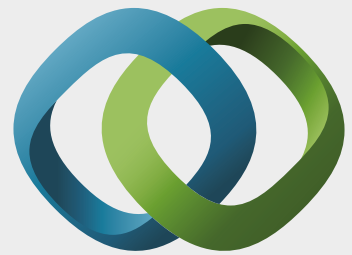

\section{Hindawi}

Submit your manuscripts at

https://www.hindawi.com
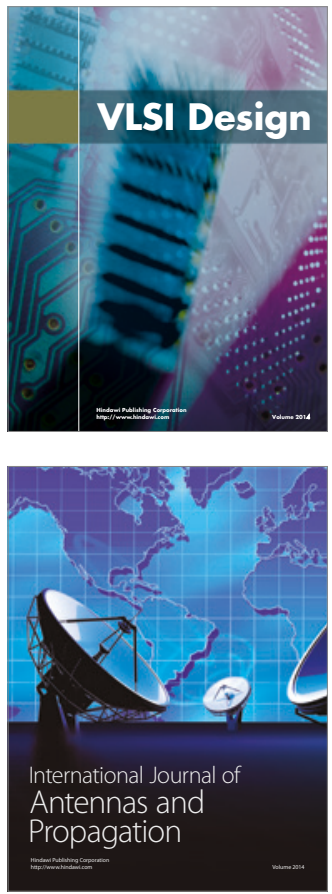

\section{Rotating}

Machinery
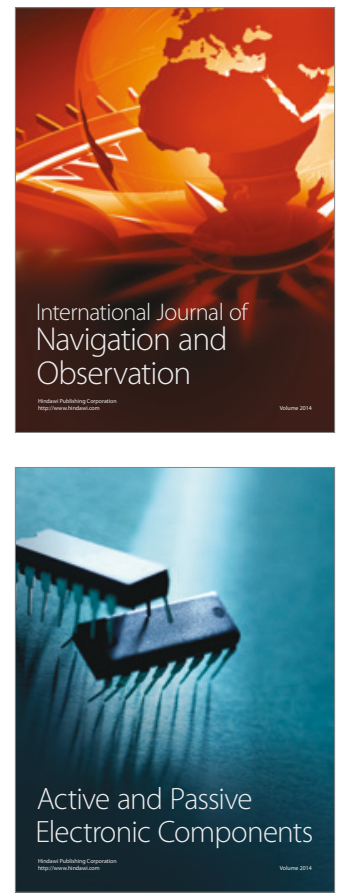
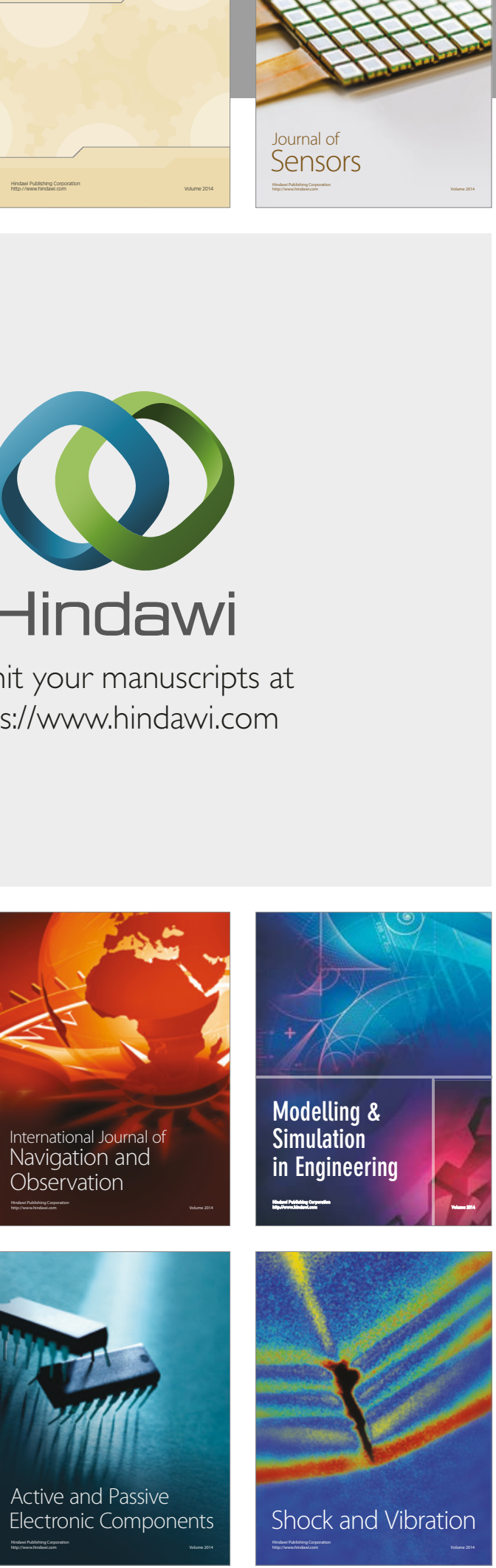
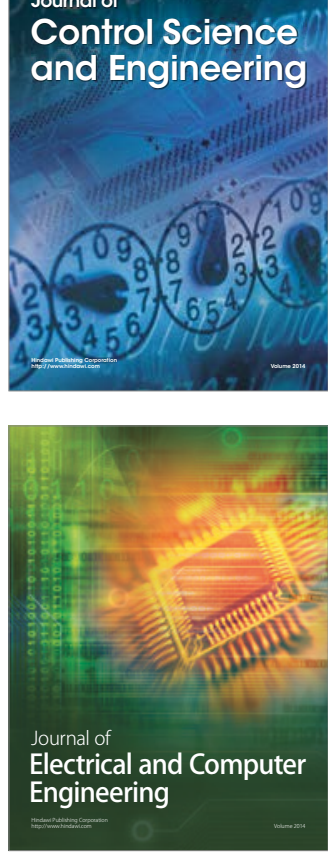

Distributed

Journal of

Control Science

and Engineering
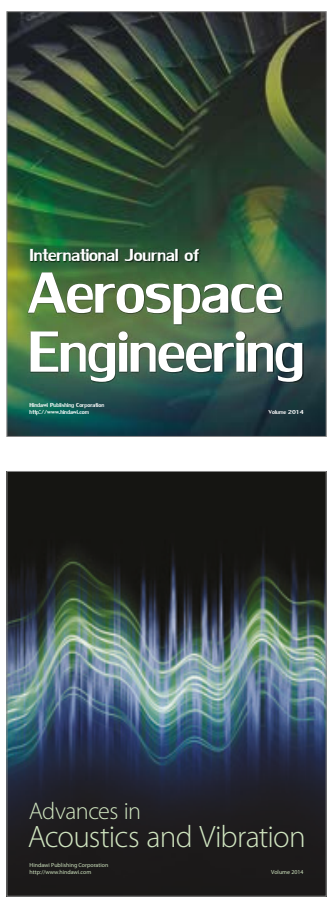

Sensor Networks 